\title{
Análisis de la morfología interna del primer molar superior mediante la técnica de diafanización
}

\author{
Analysis of the internal morphology of the maxillary first molar through the \\ diafanization technique \\ Vanessa Montesinos-Rivera (1) ${ }^{1 a}$, Priscilla Medina-Sotomayor (1) ${ }^{1 b}$, María José Sánchez-Ordóñez (1) 1a
}

\section{RESUMEN}

Objetivo: determinar la morfología interna del primer molar superior en una muestra obtenida en Cuenca-Ecuador mediante la técnica de diafanización. Materiales y métodos: en una muestra de 84 primeros molares superiores y siguiendo la clasificación de Vertucci se analizó la morfología interna de los conductos radiculares mediante la técnica de diafanización. Resultados: el $58 \%$ de la muestra presentó tres conductos radiculares, seguido por la presencia de cuatro conductos radiculares en un $38 \%$ de los casos. El conducto visible en la figura 1 (a) de la ilustración fue el más frecuente, solo se encontró un conducto en la raíz mesio vestibular. Conclusiones: en la muestra analizada existe mayor frecuencia de tres conductos radiculares con configuración tipo I.

Palabras clave: Endodoncia; Técnica de descalcificación; Diafanización; Anatomía; Primer molar superior; Ecuador. (Fuente: DeCS BIREME)

\section{ABSTRACT}

Objective: determine the internal morphology of the upper first molar in a sample obtained in Cuenca-Ecuador using the diaphanization technique. Material and Metods: $\mathrm{n}$ a sample of 84 upper first molars and following the Vertucci classification, the internal morphology of the root canals was analyzed using the diaphanization technique. Results: $58 \%$ of the sample had three root canals, followed by the presence of four root canals in $38 \%$ of the cases. The canal visible in figure 1 (a) of the illustration was the most frequent, only one canal was found in the mesiobuccal root. Conclusions: $n$ the analyzed sample, there is a higher frequency of three root canals with type I configuration.

Keywords: Endodontics; Decalcification technique; Diafanization; Anatomy; Maxillary first molar; Equator. (Source: MeSH NLM)

${ }^{1}$ Universidad Católica de Cuenca, Carrera de Odontología, Sede Azogues, Ecuador

a, Especialista en Endodoncia

b. Doctora en Odontología.

\section{Correspondencia:}

Priscilla Medina-Sotomayor

Dirección: Av. 16 de abril, Azogues, Ecuador

Correo electrónico: ipmedinas@ucacue.edu.ec
Este es un artículo de acceso abierto distribuido bajo la licencia Creative Commons Atribución 4.0 Internacional (CC BY 4.0) https://creativecommons.org/licenses/by/4.0/deed.es

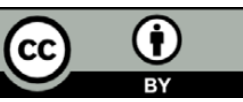

Citar como: Montesinos-Rivera V, Medina-Sotomayor $\mathrm{P}$, Sánchez-Ordóñez MJ. Análisis de la morfología interna del primer molar superior mediante la técnica de diafanización. KIRU. 2021 Jul-Sep; 18(3): 133- 139.

https://doi.org/10.24265/kiru.2021.v18n3.01 


\section{INTRODUCCIÓN}

Conocer a fondo la anatomía de las piezas dentales, es uno de los aspectos fundamentales para conseguir el éxito en la terapia endodóntica, que tiene como uno de sus principales objetivos, realizar una preparación biomecánica que englobe la mayor parte del sistema de conductos radiculares ${ }^{(1)}$.

Los primeros molares superiores presentan una compleja anatomía interna. Existe variación en la literatura con respecto al número de conductos por raíz, especialmente en la raíz mesiovestibular (MV), llamado también MV1 y el conducto palatino. Su localización es difícil, dada la presencia de un abultamiento dentinario en la pared mesial que cubre al surco sub pulpar, donde se ubica la entrada de dicho conducto. Si no se elimina esta dentinificación, el cuarto conducto puede pasar inadvertido durante el tratamiento endodóntico y provocar infecciones, o complicar una ya presente, trayendo como consecuencia un tratamiento deficiente. A este conducto se lo denomina mesio vestibular 2 (en adelante: MV 2) o mesio palatino (de aquí en más: MP) ${ }^{(2)}$.

Existe mucha información científica sobre la anatomía de conductos radiculares realizados en diversas poblaciones, lamentablemente no se encontró ningún antecedente en muestras ecuatorianas.

Entre los métodos de investigación empleados para estudiar la anatomía dental mencionaremos las tomas radiográficas en angulaciones, los cortes histológicos, la reconstrucción tridimensional asistida por ordenador, la microscopía de resonancia magnética, y también, la técnica de diafanización. Esta última, cuenta entre sus beneficios el proporcionar imágenes que permiten (dentro del estudio morfológico de las piezas) el análisis exhaustivo de la anatomía pulpar. Por esta técnica se logra visualizar tridimensionalmente tanto la cámara pulpar como los conductos radiculares, preservando internamente su forma original, sus anastomosis y la conservación de la muestra por períodos prolongados ${ }^{(3)}$.

Actualmente se plantean nuevos métodos que incluyen la Tomografía computarizada (TC); Tomografía computarizada de haz cónico (CBCT según sus siglas en idioma inglés), Microtomografía computarizada (MCT según sus siglas en idioma inglés) que permiten la reconstrucción morfológica interna y externa de la pieza dental ${ }^{(4-8)}$.

Las complejidades internas del conducto radicular están determinadas genéticamente $\mathrm{y}$ tienen una importancia definida en la antropología, por lo que es necesario identificar las morfologías del conducto radicular de diferentes poblaciones. Por la presencia de estas estructuras, la configuración morfológica de los conductos radiculares ha sido clasificada por algunos autores, destacando a Weine quien determina cuatro categorías para la presencia de 1 o 2 conductos en la misma raíz y la más utilizada a nivel mundial, es la de Vertucci. (Figura 1) $^{(9-11)}$.

El objetivo del presente estudio es investigar la morfología interna del primer molar superior mediante la técnica de diafanización en una muestra obtenida en Cuenca-Ecuador para así proporcionar a los especialistas en endodoncia de la región mayor precisión en los tratamientos, logrando resultados más predecibles.

\section{MATERIAL Y METODOS}

El presente estudio in vitro se realizó en la Universidad de Cuenca, Ecuador. Los dientes extraídos fueron obtenidos de las diferentes unidades de salud odontológica de la mencionada ciuda, previo consentimiento del Ministerio de Salud. Todos los procedimientos se realizaron según las normas establecidas por esta entidad gubernamental con la correspodiente aprobación del comité de ética. La naturaleza de la muestra imposibilitó determinar la edad y el género. Se obtuvo un total de 84 primeros molares superiores que cumplieron con los criterios de selección. Fueron determinados como criterios de inclusión: primeros molares superiores permanentes que presenten integridad radicular y ápices cerrados y como criterio de exclusión: piezas dentales que presenten reabsorciones radiculares externas 0 internas, fracturas radiculares, caries radiculares, formación radicular incompleta, con previa manipulación de los conductos radiculares o con tratamientos de endodoncia previos.

Las muestras fueron previamente desinfectadas en hipoclorito de sodio al 5\% por 24 horas, luego se realizó una limpieza manual con una cureta 13-14 Gracey (HuFriedy, USA) para la eliminar residuos orgánicos adheridos a la superficie dental y lavando posteriormente con agua corriente. Las coronas dentales fueron 
seccionadas hasta el límite amelocementario con discos de carborundo. El piso cameral fue examinado con un explorador endodóntico DG16 (HuFriedy Mfg. Co., Chicago, IL, USA). Localizados los orificios de entrada hacia los conductos palatinos y disto vestibulares, se permeabilizó para llegar al foramen apical hasta una lima de acero inoxidable $\mathrm{K}$ \#8 y \#10 (Maillefer Ballaigues, Suiza). Para la raíz mesio vestibular se utilizó una lima $\mathrm{K} \# 6$, \#8 y \#10 (Maillefer Ballaigues, Suiza) sin movimientos de limado, luego con fresas LN (Maillefer Ballaigues, Suiza) se eliminó la proyección dentinaria que cubre el orificio de entrada hacia el conducto radicular MV2.

Todo el procedimiento se realizó con irrigación continúa empleando hipoclorito de sodio al $2 \%$ para eliminar la pulpa dental presente. El remanente orgánico restante fue removido del interior por la inmersión de hipoclorito de sodio al $5 \%$ durante 24 horas, luego las muestras fueron lavadas con agua corriente por otras 24 horas antes de ser almacenada en solución salina.
El protocolo de diafanización se realizó con ácido nítrico al $5 \%$ durante 3 días cambiando diariamente la solución (se mantuvo a temperatura ambiente).

Luego la muestra fue lavada en agua corriente por 4 horas, para posteriormente aplicar concentraciones ascendentes de etanol por 30 minutos cada una desde el 20 al $96 \%$. Luego se colocaron en salicilato de metilo. Para evidenciar la anatomía interna de los conductos radiculares, se inyecto tinta china, el exceso fue eliminado por presión negativa desde el extremo apical del diente con el uso de una punta de succión adaptada al sistema de aspiración de la unidad.

Las muestras fueron divididas aleatoriamente en dos grupos para ser examinadas por dos observadores independientes al estudio, previamente calibrados (Kappa 0,72), quienes con la ayuda de un microscopio óptico a x12 de aumento (JO Soarez, Rio de Janeiro, Brasil) procedieron al análisis de la morfología interna utilizando la clasificación de Vertucci. (Figura 1).
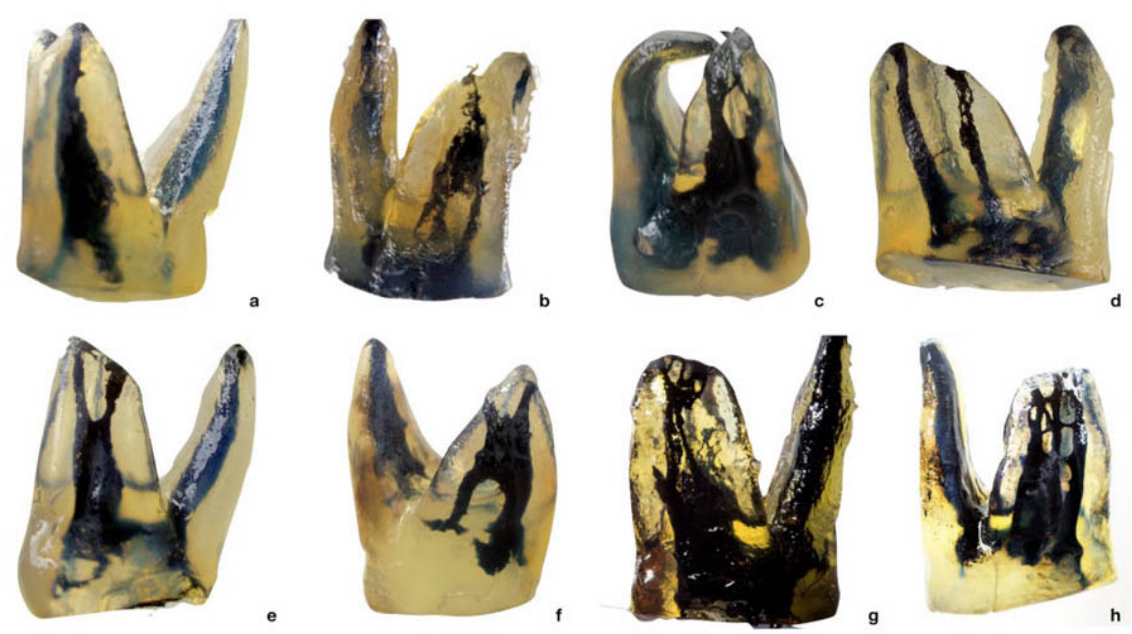

Figura 1: Clasificación de Vertucci según el tipo de conducto radicular en el primer molar superior mediante la técnica de diafanización. a) Conducto Tipo I: un solo conducto hasta nivel apical, b) Conducto Tipo II: inician dos conductos por separado y se unen a nivel apical, c) Conducto Tipo III: el conducto se divide en dos para luego fusionarse y terminar en uno solo, d) Conducto Tipo IV: dos conductos inicialmente separados van a lo largo del conducto y terminan de manera independiente, e) Conducto Tipo V: un conducto se divide en apical en dos conductos independientes, f) Conducto Tipo VI: dos conductos individuales se fusionan, en su recorrido se dividen nuevamente y terminan como dos conductos individuales, g) Conducto Tipo VII: El conducto sale de la cámara pulpar, se divide, luego se une y terminan dividido en dos conductos diferentes y h) Conducto Tipo VIII: Tres conductos separados que van desde la cámara pulpar hasta el ápice. 


\section{RESULTADOS}

El total de primeros molares superiores analizados fue de 84, encontrando un solo conducto en la raíz palatina y disto vestibular en el $98,8 \%$ y $96,4 \%$ de la muestra respectivamente, mientras que en la raíz mesio vestibular (tabla 1): 49 raíces presentaron un conducto $(58.3 \%)$, en 32 raíces se encontraron 2 conductos $(38.1 \%)$ y en 3 raíces fueron hallados 3 conductos $(3.6 \%)$.
Según la clasificación de Vertucci, el conducto tipo I fue la más prevalente en la raíz palatina y disto vestibular con un 98.8 y $96.4 \%$ respectivamente. En la raíz mesio vestibular el conducto tipo I se encontró en 26 piezas (31\%) seguido por el tipo $\mathrm{V}$ con $\mathrm{u}$ total de 21 piezas (25\%). (Tablas 2-4) (Fig. 1). En cuanto al conducto MV2, se encontró presente en un total de 35 piezas $(41.7 \%)$.

Tabla 1. Número de conductos mesio vestibulares del primer molar superior

\begin{tabular}{ccc}
\hline $\begin{array}{c}\text { Número de conductos mesio } \\
\text { vestibulares }\end{array}$ & $\mathbf{n}$ & $\mathbf{\%}$ \\
\hline $\mathbf{1}$ & 49 & 58.3 \\
$\mathbf{2}$ & 32 & 38.1 \\
$\mathbf{3}$ & 3 & 3.6 \\
\hline TOTAL & $\mathbf{8 4}$ & $\mathbf{1 0 0}$ \\
\hline
\end{tabular}

Tabla 2. Tipo de conducto palatino del primer molar superior según la clasificación de Vertucci.

\begin{tabular}{ccc}
\hline Tipo de conducto palatino & $\mathbf{n}$ & $\%$ \\
\hline I & 83 & $98.8^{*}$ \\
III & 1 & 1.2 \\
\hline TOTAL & $\mathbf{8 4}$ & $\mathbf{1 0 0}$ \\
\hline
\end{tabular}

Tabla 3. Tipo de conducto disto vestibular del primer molar superior según la clasificación de Vertucci.

\begin{tabular}{ccc}
\hline $\begin{array}{c}\text { Tipo de conducto disto } \\
\text { vestibular }\end{array}$ & $\mathbf{n}$ & $\%$ \\
\hline I & 81 & $96.4^{*}$ \\
III & 2 & 2.4 \\
\hline TOTAL & 1 & 1.2 \\
\hline
\end{tabular}


Tabla 4. Tipo de conducto mesio vestibular del primer molar superior según la clasificación de Vertucci.

\begin{tabular}{ccc}
\hline $\begin{array}{c}\text { Tipo de conducto mesio } \\
\text { vestibular }\end{array}$ & $\mathbf{n}$ & \% \\
\hline I & 26 & $31^{*}$ \\
II & 14 & 16.7 \\
III & 1 & 1.2 \\
IV & 12 & 14.3 \\
V & 21 & 25 \\
VI & 6 & 7.1 \\
VII & 1 & 1.2 \\
VIII & 1 & 1.2 \\
otros & 2 & 2.4 \\
\hline TOTAL & $\mathbf{8 4}$ & $\mathbf{1 0 0}$ \\
\hline
\end{tabular}

\section{DISCUSIÓN}

Un tratamiento endodóntico exitoso se logra cuando todos los conductos radiculares han sido localizados, desbridados, desinfectados y obturados completamente. Entre las razones de fracaso en estos tratamientos se incluye una desinfección incompleta y obturación inadecuada, por lo tanto, es indispensable conocer las diversas variaciones anatómicas que se puedan dar en los conductos radiculares ${ }^{(1)}$. La morfología del primer molar permanente ha sido estudiada por diversos investigadores ya que es el primero en erupcionar. Por lo tanto, es también el más propenso a desarrollar caries dental, convirtiéndose en el que más frecuentemente se trata endodónticamente. La variación de la morfología de estos dientes ha sido estudiada en varias poblaciones encontrando diferencias en cada una de ellas lamentablemente la población ecuatoriana no tiene ningún antecedente de estudio recalcando la pertinencia de centrar estudios similares en esta población.

Diferentes técnicas han sido usadas para la evaluación de la morfología de los conductos radiculares. Recientemente, el uso de la microtomografía computada (MCT) y la tomografía computada de haz cónico (o CBCT) han sido consideradas excelentes para este propósito.

La MCT es la que proporciona la mayor precisión y la más alta resolución. La CBCT, además de utilizarse para estudios de laboratorio, se utiliza para el diagnóstico en la práctica clínica, siendo una gran ayuda para este fin de evaluación morfológica ${ }^{(12)}$. Sin embargo, para su indicación se debe de tener en cuenta la dosis de radiación a la que se exponen los pacientes ${ }^{(12,13)}$. La técnica de diafanización (clarificación o descalcificación) utilizada en nuestra investigación tiene un valor considerable para el estudio de la morfología de los conductos radiculares. Con ella se obtiene una vista tridimensional del interior de la pieza dental, revelando conductos transversales, deltas apicales y otras complejidades del conducto radicular ${ }^{(1,12,14)}$, no requiriendo la utilización de aparatos complejos. La diafanización es, además, una técnica de bajo costo (12,14), constituyendo una importante herramienta para la docencia. A pesar de las grandes ventajas que presenta, posibles complicaciones durante su realización deben ser consideradas. Entre ellas se destacan: la dificultad de determinar el grado de desmineralización de las piezas 
dentales una deshidratación incompleta y la posible opacidad resultante ${ }^{(1,4,9,14)}$.

En esta investigación se utilizó tinta china para culminar la diafanización de la pieza dental. La tinta fue introducida en el interior del conducto para su mejor visualización. Ello se debe al tamaño de las partículas de esta tinta y a su capacidad de penetración ${ }^{(1)}$.

Malentacca y Ordinola en 2015 y 2017 respectivamente, al han modificado la técnica de aclaramiento: en su variación no se realiza la descalcificación; con esto se logra el objetivo de mantener una dureza similar en la dentina, permitiendo también estudios de instrumentación (12, 14). Para el objetivo de nuestro trabajo, la técnica original (a pesar de su antigüedad) aporta un gran recurso científico que mantiene su significación en el campo de la endodoncia.

La clasificación es la más aceptada mundialmente debido a su relevancia clínica es la de Vertucci. Las ocho configuraciones radiculares descritas por el autor fueron identificados en los primeros molares superiores analizados en el presente trabajo. Estos hallazgos están en concordancia con otros estudios de diferentes poblaciones ${ }^{(12,15-21)}$.

La mayoría de las piezas dentales presentaron tres conductos radiculares (58\%) solo en un $38 \%$ se evidenciaron cuatro, lo que concuerda con estudios en poblaciones americanas y orientales (22-23), lo que indica una alta prevalencia de esta forma anatómica. La frecuencia de cinco conductos radiculares fue muy baja ${ }^{(24)}$.

La prevalencia de MV2 en el primer molar superior ha sido investigado extensivamente, pero con variaciones relacionadas con la raza, el género y la edad de la población. ${ }^{(19-23)} \mathrm{En}$ el presente estudio se encontró una frecuencia del $41 \%$, siendo menor que en previos estudios realizados en asiáticos, americanos, hispanos y otros, cuya frecuencia se encontraba en $90 \%$, esto puede deberse a las diferencias en la metodología de evaluación de la pieza dental, la etnia y la edad a la que corresponde la muestra, siendo imposible determinarla ${ }^{(22,25)}$.

Los conductos tipo I, II y IV han sido reportados como los más comunes en las raíces mesio vestibulares, sin importar la etnia, siendo el más frecuente el tipo I $(18,23,26)$. El fallo en los tratamientos de conductos ha sido relacionado a una preparación incompleta de los conductos debido a la compleja anatomía de los mismos. EI conocimiento previo de la anatomía interna de los primeros molares superiores puede evitar errores como falla en la instrumentación, especialmente en una población que no ha sido estudiada previamente. Dentro de las limitaciones de este estudio in vitro, en una muestra de la población ecuatoriana, se observo que en el primer molar superior, la frecuencia más alta es la presencia de tres conductos radiculares con configuración tipo I, pero con alta prevalencia del conducto MV2 o varios conductos en la raíz mesio vestibular que pueden derivar en procedimientos endodónticos de alta complejidad. Los odontólogos deben ser conscientes de la complejidad de la anatomía del conducto radicular utilizando la información científica más reciente y confiable para lograr resultados de tratamiento favorables.

Contribución de autoría: PMS. Análisis e interpretación de datos. VMR. concepción y diseño del trabajo, aporte de material de estudio. MJSO Diseño del trabajo. Los autores redactaron, realizaron y aprobaron la versión final del manuscrito.

Fuentes de financiamiento: autofinanciada.

Conflicto de Interés: Los autores declaran no tener conflicto de interés.

\section{REFERENCIAS BIBLIOGRÁFICAS}

1. Katge F, Wakpanjar MM. Root canal morphology of primary molars by clearing technique: an in vitro study. J Indian Soc Pedod Prev Dent. 2018;36(2):151-157.

2. Degerness RA, Bowles WR. Dimension, Anatomy and Morphology of the Mesiobuccal Root Canal System in Maxillary Molars. J Endod.2010;36(6):985-9.

3. Omer OE, Al Shalabi RM, Jennings M, Glennon J, Claffey NM. A comparison between clearing and radiographic techniques in the study of the rootcanal anatomy of maxillary first and second molars. Int Endod J.2004;37(5):291-6.

4. Michetti J, Maret D, Mallet J, Diemer F. Validation of Cone Beam Computed Tomography as a Tool to Explore Root Canal Anatomy. Journal of Endodontics. 2010;36(7):1187-1190.

5. Peters O.A. Current challenges and concepts in the preparation of root canal systems: a review. J Endod 2004;30(8):559-67.

6. Gopikrishna V, Bhargavi N, Kandaswamy D. Endodontic management of a maxillary first molar with a single root and a single canal diagnosed with the aid of spiral CT: a case report. J Endod.2006;32(7):687-691.

7. Celikten B, Orhan K, Aksoy U, Tufenkci P, Kalender A, Basmaci F, Dabaj P. Cone-beam CT 
evaluation of root canal morphology of maxillary and mandibular premolars in a Turkish Cypriot population. BDJ Open.2016;29(2):15006.

8. Hajihassani N, Roohi N, Madadi K, Bakhshi M, Tofangchiha M. Evaluation of Root Canal Morphology of Mandibular First and Second Premolars Using Cone Beam Computed Tomography in a Defined Group of Dental Patients in Iran. Scientifica (Cairo).2017;2017:1504341.

9. Bagherian A, Kalhori KA, Sadeghi M, Mirhosseini F, Parisay I. An in vitro study of root and canal morphology of human deciduous molars in an Iranian population. J Oral Sci 2010;52(3):397-403.

10. Liu JF, Dai PW, Chen SY, Huang HL, Hsu JT, Chen WL, et al. Prevalence of 3-rooted primary mandibular second molars among Chinese patients. Pediatr Dent.2010;32(2):123-6.

11. Vertucci F. J. Root canal anatomy of the human permanent teeth. Oral Surgery, Oral Medicine, Oral Pathology. 1984;58(5):589-599

12. Ordinola-Zapata R, Bramante CM, Versiani MA, Moldauer BI, Topham G. et al. Comparative accuracy of the clearing technique, CBCT and Micro-CT methods in studying the mesial root canal configuration of mandibular first molars. Int Endod J. 2017;50(1):90-96.

13. Chourasia HR, Meshram G.K, Warhadpande M, Dakshindas D. Root Canal Morphology of Mandibular First Permanent Molars in an Indian Population. I J Dent.2012;2012:745152.

14. Malentacca A., Lajolo C. A new technique to make transparent teeth without decalcifying: Description of the methodology and microhardness assessment. Ann Anat. 2015; 197:11-5

15. Yeun K, Seok-Woo C, Jong-Ki LA, I-Ping C, Blythe $K$. et al. A micro-computed tomography study of canal configuration of multiple-canalled mesiobuccal root of maxillary first molar. Clin Oral Investig. 2013;17(6):1541-6.

16. Robertson, I. Leeb J, McKee M, Erich Brewer E. A clearing technique for the study of root canal systems. J Endod.1980;6(1):421-4.

17. Ezoddini F, Mohammadi Z, Tabrizizadeh M. Root Canal Morphology of Human Mandibular Incisors in Yazd Province. Dental Research Journal.2006;3(1):1- 4.
18. Venturi M, Prati C, Capelli G, Falconi M, Breschi L. A preliminary analysis of the morphology of lateral canals after root canal filling using a toothclearing technique. Int Endod J.2003;36(1):54-63.

19. Al-Shehri S, Al-Nazhan S, Shoukry S, Al-Shwaimi E, Al-Sadhan R, Al-Shemmery B. Root and canal configuration of the maxillary first molar in a Saudi subpopulation: A cone-beam computed tomography study. Saudi Endod. J.2017;7(2):6976.

20. Kharaisat A, Smadi L. Canal configuration in the mesio-buccal root of maxillary first molar teeth of a Jordanian population. Aust Endod J. 2007;33(1):13-7.

21. Kim Y, Lee SJ, Woo J. Morphology of maxillary first and second molars analyzed by cone-beam computed tomography in a Korean population: Variations in the number of roots and Canals and the incidence of fusión. J Endod 2012;38(8):10638.

22. Guo J, Vahidnia A, Sedghizadeh P, Enciso R. Evaluation of root and canal morphology of maxillary permanent first molars in a North American population by cone-beam computed tomography. J Endod.2014;40(5):635-9.

23. Silva EJ, Nejaim $Y$, Silva AI, Haiter-Neto $F$, Zaia $A A$, Cohenca N. Evaluation of root canal configuration of maxillary molars in a Brazilian population using cone-beam computed tomographic imaging: An in vivo study $\mathrm{J}$ Endod.2014;40(2):173-6.

24. Kakkar P, Singh A. Maxillary first molar with three mesiobuccal canals confirmed with spiral computer tomography. J Clin Exp Dent.2012;4(4):256-9.

25. Lee JH, Kim KD, Lee JK, Park W, Jeong JS, Lee $\mathrm{Y}$, et al. Mesiobuccal root canal anatomy of Korean maxillary first and second molars by conebeam computed tomography. Oral Surg Oral Med Oral Pathol Oral Radiol Endod 2011;111(6):78591.

26. Reis AG, Grazziotin-Soares R, Barletta FB, Fontanella VR, Mahl CR. Second canal in mesiobuccal root of maxillary molars is correlated with root third and patient age: A cone-beam computed tomographic study. J Endod 2013;39(5):588-92

\footnotetext{
Priscilla Medina-Sotomayor

ORCID (D) https://orcid.org/0000-0002-8117-8550

ipmedinas@ucacue.edu.ec

Vanessa Montesinos-Rivera

ORCID (1) http://orcid.org/0000-0001-7991-366X

mmmontesinosr@ucacue.edu.ec

María José Sánchez-Ordóñez

ORCID https://orcid.org/0000-0001-8214-382X

mjsanchezo@ucacue.edu.ec
} 